\title{
Supplementing wind turbine pitch control with a trailing edge flap smart rotor
}

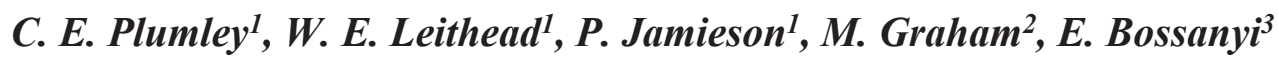 \\ ${ }^{1}$ University of Strathclyde, United Kingdom, charles.plumley@strath.ac.uk \\ ${ }^{2}$ Imperial College London, United Kingdom \\ ${ }^{3}$ DNV GL, United Kingdom
}

Keywords: smart rotor wind turbine control

\begin{abstract}
Placement of additional control devices along the span of the wind turbine blades is being considered for multi-MW wind turbines to actively alter the local aerodynamic characteristics of the blades. This smart rotor approach can reduce loads on the rotor due to wind field non-uniformity, but also, as presented in this paper, can supplement the pitch control system. Rotor speed and tower vibration damping are actively controlled using pitch. By supplementing the speed control using smart rotor control, pitch actuator travel is reduced by $15 \%$, pitch rates by $23 \%$, and pitch accelerations by $42 \%$. This is achieved through filtering the pitch demand such that high frequency signals are dealt with by the smart rotor devices while the low frequency signal is dealt with by pitching the blades. It is also shown that this may be achieved while also using the smart rotor control for load reduction, though with reduced effectiveness. This shows that smart rotor control can be used to trade pitch actuator requirements as well as load reductions with the cost of installing and maintaining the distributed devices.
\end{abstract}

\section{Introduction}

Global warming, energy security and the increasing volatility in fossil fuel prices have encouraged investment in renewable forms of electricity generation, and in many countries has led to strict renewable targets for the deployment of renewables [1]. Wind energy is one such renewable form of generation that has low greenhouse gas emissions and is an indigenous and sustainable resource. The wind industry however needs to compete economically with other sources of electricity. It is therefore essential to reduce the cost of energy from wind not only to benefit the consumer, but to increase the industry's competiveness and so assure its long term survival.

To bring down the cost of energy the size of wind turbines has increased considerably in the past few decades and offshore, where unit costs such as foundations, construction and connections are high, the trend is to even larger wind turbines [2]. The increasing size of wind turbines comes with its problems though. The optimum size of the turbines is naturally limited by scaling laws, as while energy capture scales with the square of the rotor diameter, mass scales approximately to the power of three [3], but the size is also limited by loads on the rotor. In particular, the non-uniformity of the wind field encountered by the rotor due to wind shear and turbulence causes large cyclic loadings. These loads increase the material requirements and so need mitigating to allow a reduction in the cost of energy [e.g. 4].

Control is an essential component of modern multi-MW wind turbine design. Not only is it used to optimise energy capture and maintain rated power, but also to minimise loads. This is done in part through avoiding resonant frequencies and active damping [5]. With advanced control techniques it is also possible to reduce the loads on the rotor and wind turbine in general through actively adjusting the rotor aerodynamics to account for variations in the wind field across the rotor.

Smart rotor control involves placement of active devices on the blades capable of changing the local aerodynamics. Historically tip devices have been used for over-speed protection as an alternative to pitch control and often these were passively activated, before being superseded by full span pitch control. The modern devices under consideration for the smart rotor are actively controlled, and although able to respond rapidly to the changing environment, typically lack the ability to fully replace pitch control $[6,7]$. For example trailing edge flaps, examined in this work, are unable to offer full controllability of rotor speed across all wind speeds, as the devices saturate at a certain angle. Nevertheless, the smart rotor is capable of reducing loads as effectively as individual pitch control [8].

Supplementing pitch control with a smart rotor control has been demonstrated for load reductions by combining individual pitch and the smart rotor controls, with low frequency loads targeted by the individual pitch control and high frequencies by the smart rotor control. This has resulted in better overall load reductions than either one individually, [9]. However, upgrading the pitch actuators and controller gains could result in similar enhancements in load reduction and a true comparison needs to take account of the choice between either upgrading the pitch actuators or implementing a smart rotor [8]. This is the first study to look at using the smart rotor to supplement the main collective pitch control mechanism for rotor speed control, maintaining controllability and reducing demands on the pitch actuator. 


\section{Method}

DNV GL's Bladed [10], a wind turbine simulation software package used in the design and certification of wind turbines, is used to model, simulate and assess the performance of the supplementary smart rotor control strategies proposed here.

The NREL 5MW conceptual wind turbine described in [11] was modelled in Bladed. This is a well-documented conceptual wind turbine based on the REpower 5MW and other large scale wind turbines available at the time of its design. It is widely used in control research and so eases comparisons with other work, and is also of a size where advanced load reduction techniques become desirable. It is an upwind variable speed pitch regulated machine with an operating range of $4-24 \mathrm{~m} / \mathrm{s}$. The maximum generator speed is $1173.7 \mathrm{rpm}$, which corresponds to a rotor speed of $12.1 \mathrm{rpm}$ or $1.267 \mathrm{rad} / \mathrm{s}$. Rated wind speed is $11.4 \mathrm{~m} / \mathrm{s}$.

The focus of this paper is the advantages that supplementary smart rotor control can give and not on the specific implementation of one type of smart rotor technology. Despite this, to make the study realistic, the NREL 5MW conceptual wind turbine model is adjusted to include trailing edge flaps with properties similar to those of the Sandia demonstration plant [12]. However, the control methods used are not limited to use with only trailing edge flaps.

The trailing edge flaps are similar to the ailerons found on aircraft wings. They are here assumed to make up $20 \%$ of the blade chord width, $20 \%$ of the blade span and centred at $87 \%$ of the blade length, such that there is one chord width between the end of the blade and the start of the flap. The flaps are limited to a maximum deflection angle of 20 degrees. The flap rates of the Sandia demonstration plant are high, averaging $200 \%$ s and peaking at $330 \%$ second. The flaps simulated are not rate or acceleration limited in the model, but the maxima reached during the simulations are shown for reference and do not exceed those of the demonstration plant.

Rather than the control described by Jonkman in the description of the NREL 5MW wind turbine, the baseline control used is that of the UpWind wind turbine described in [13]. This is a state-of-the-art variable speed pitch controller. Below rated, torque control is used to track the optimum tip speed ratio, while above rated power, where pitch control is active, power is held constant through adjustment of the torque, and rotor speed is held constant using a PI-based pitch control mechanism with varying gain. The rotor speed control mechanism is described in section 3, with the supplementary control design described in section 4. Results of the supplementary control are in section 5, and a consolidated DQ-axis smart rotor control with supplementary speed control is considered in section 6 .

\section{Rotor speed control}

Rotor speed above rated is controlled by adjusting the collective pitch angle of the blades. To reduce the aerodynamic torque on the rotor the blades are pitched towards feather, reducing the angle of attack and thus lowering the lift produced. This is done using a Proportional and Integral (PI) controller based on rotor speed error, and additionally a term to take account of the difference between actual power and rated power, to encourage pitching in rising wind speeds in the region just below rated. A gain schedule $(\mathrm{GK})$ is also used to account for the fact that at higher wind speeds less pitch action is required to achieve the same controllability [14]. The rotor speed control system is shown in figure 1.

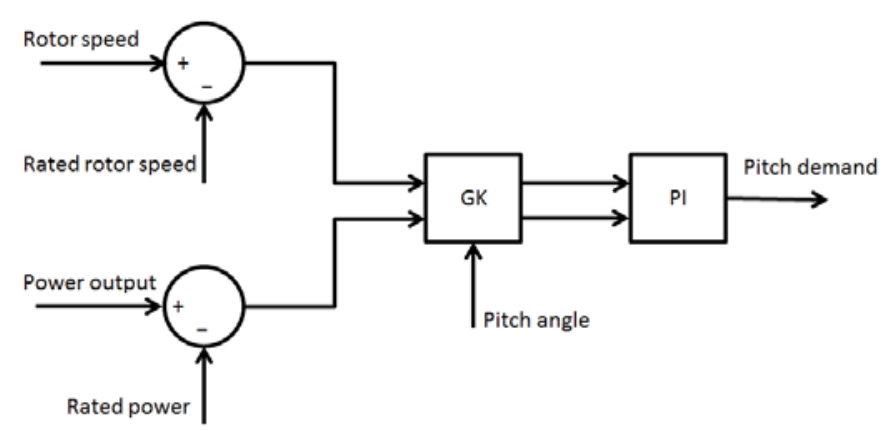

Figure 1 Rotor speed control system diagram

The two controllers share the same integrator and anti-windup limits, which are imposed on the integral in the form of minimum and maximum pitch angles and maximum rates. For the rotor speed error a proportional gain of 0.0135 and integral gain of 0.00453 are used and for the power error a proportional and integral gain of $10^{-7}$ and $5 \times 10^{-8}$ are used respectively. The gain scheduling uses the current pitch angle to adjust the gain and is of the form GK $=1 /(1.0+\theta / 12.5)$, with the pitch angle, $\theta$, in degrees, and a minimum gain imposed of 1/3.5. This is described in detail in reference [13].

\section{Supplementary control design}

The supplementing of the pitch speed control by the smart rotor is done through splitting the demanded pitch angle from the PI speed controller based on frequency. High frequency variations are controlled by the smart rotor, which is considered more than capable of rapid response, and low frequency variations are left to be controlled by the pitch. Single pole low and high pass filters are found to be adequate for this role, implemented as recursive filters. A series of filter cut-off frequencies are trialled to determine the optimum cut-off frequencies for the supplementary control, taking account of the impact on the pitch actuator, flap actuator and rotor speed.

For these results two 10-minute runs using Kaimal 3D turbulent wind fields are run for each wind speed from 10 to $24 \mathrm{~m} / \mathrm{s}$ in $2 \mathrm{~m} / \mathrm{s}$ intervals, and the maximum pitch rates and accelerations, flap deflections and rotor speed variations are 
found for each different cut-off frequency. This allows an initial analysis of what effect the supplementary control has on the wind turbine and a discovery of what filter cut-off frequency is preferable.
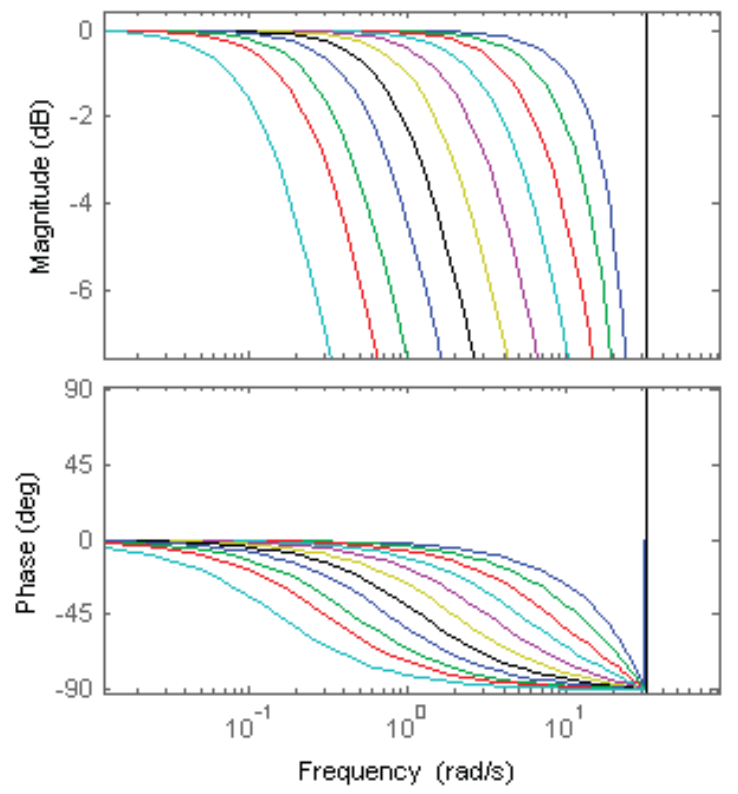

Figure 2 Bode plot of low pass discrete filters with increasing cut-off frequencies

At high frequencies the filters are pushed to their limits due to the $10 \mathrm{~Hz}$ sampling rate of the pitch controller, as seen in figure 2. However, at these high cut-off frequencies the benefit of supplementing the pitch control with the smart rotor is already diminished, and so high cut-off frequency filters are not too important.

The results of supplementing collective pitch control with smart rotor control using a variety of filters can be seen in figures 3 to 6 . In figures 3 and 4 it can be seen that as the cutoff frequency of the filters is increased, maximum pitch rates and accelerations approach those of the baseline collective pitch controlled case where the supplementary smart rotor speed control is inactive. This is to be expected as at high cutoff frequencies the majority of control is required below this frequency and so is done by the pitch control alone. This can also be seen in figure 5 as the flap motion decreases with increased filter cut-off frequency.

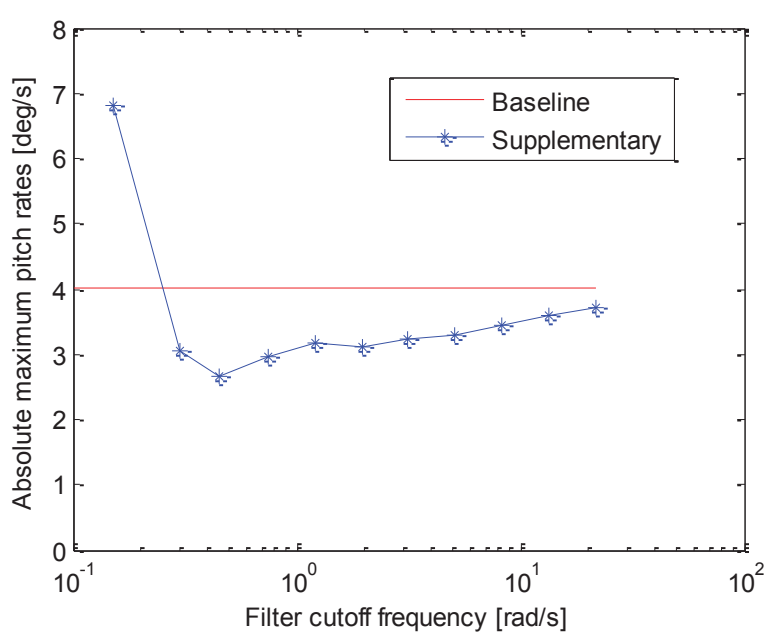

Figure 3 Maximum pitch rates across all above rated wind speeds

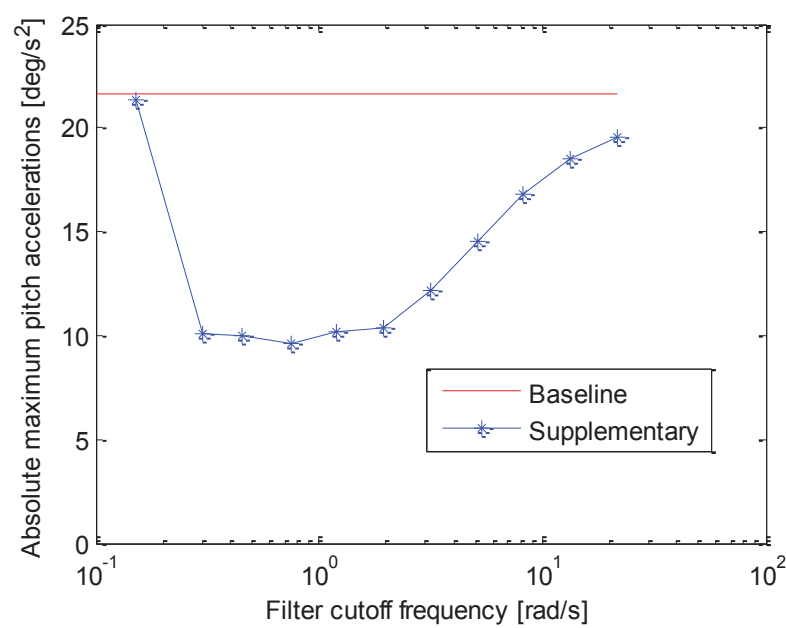

Figure 4 Maximum pitch accelerations across all above rated wind speeds

Setting the cut-off frequency too low also causes problems, again as seen in increases in the maximum pitch rates and accelerations, figures 3 and 4 . This occurs because the flap actuator saturates, as can be seen in figure 5 which portrays the maximum flap angle reached during the simulations. When this occurs controllability is lost, which also leads to larger oscillations in rotor speed, as can be seen in figure 6. It is therefore clear from this result that that trailing edge flaps are not capable of fully supplanting pitch control as they lack full controllability due to saturation at \pm 20 degrees. 


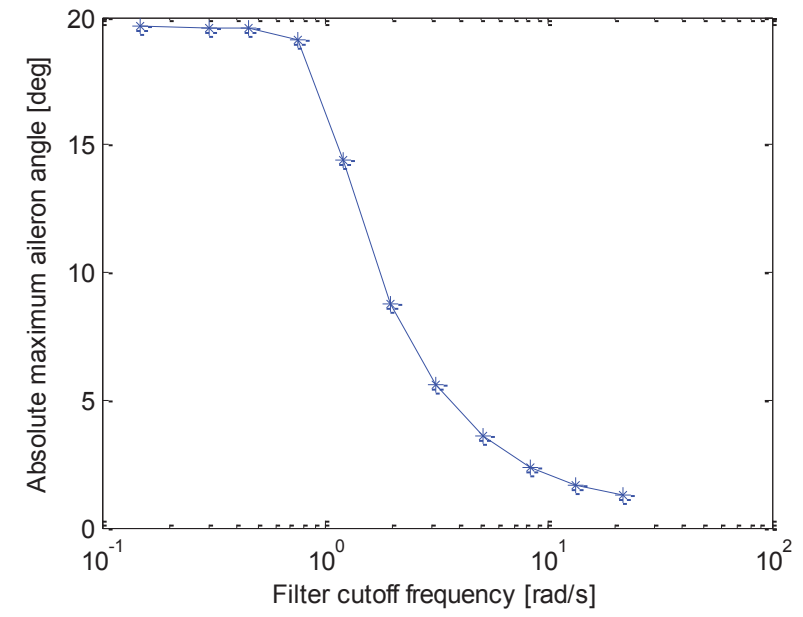

Figure 5 Maximum flap angles reached across all above rated wind speeds

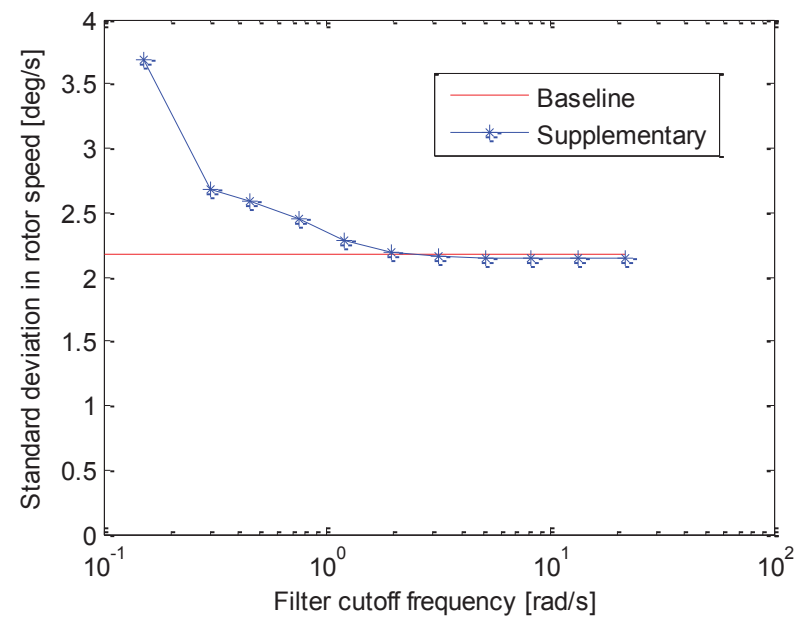

Figure 6 Mean standard deviation in rotor speed across all above rated wind speeds

These results suggest a cut-off frequency of $1.95 \mathrm{rad} / \mathrm{s}$ would be optimal, because this maintains the same rotor speed stability as the baseline case, while achieving the highest possible benefit for the pitch actuator rates and accelerations. The maximum flap angle reached is also less than half the value at which the flap saturates.

\section{Results with optimal filter}

To analyse this specific case in more detail the IEC 61400 standard is used for a class IIB turbine [15]. Six runs at each wind speed from 10 to $24 \mathrm{~m} / \mathrm{s}$ in $2 \mathrm{~m} / \mathrm{s}$ steps are used; below $10 \mathrm{~m} / \mathrm{s}$ no pitch action occurs as the wind turbine remains below rated so these simulations are the same as the base case.

The maximum flap deflection across all runs is 9.3 degrees, while reducing the maximum pitch acceleration by $42 \%$ and the maximum pitch rate by $23 \%$, and the travel by $15 \%$. The reduction in pitch rates and accelerations are independent of wind speed, as can be seen in figures 7 and 8 . The reduction in travel on the other hand is a function of wind speed, with higher gains to be had at higher wind speeds, figure 9. The trends are shown by the red line on the graphs.

The maximum flap rates and accelerations for the $1.95 \mathrm{rad} / \mathrm{s}$ cut-off frequency across all wind speeds were 23.9 degrees/s and 190.9 degrees $/ \mathrm{s}^{2}$, which is significantly below the rates and accelerations of the Sandia demonstration plant [12], however larger actuators would be required for this larger wind turbine and so achieving the same characteristics would be challenging.

Supplementing the main pitch control mechanism with smart rotor control is clearly feasible from this investigation, and allows a reduction in the pitch actuator requirements. Whether this is worth doing depends on the trade-off between the pitch actuator cost and maintenance requirements, and those of the distributed actuators as the duty changes, which is beyond the scope of the paper. The smart rotor will likely need to offer something more than just a reduction in the pitch actuator requirements though. The case where the smart rotor reduces loads as well as the pitch actuator requirements through supplementing speed control is therefore considered.

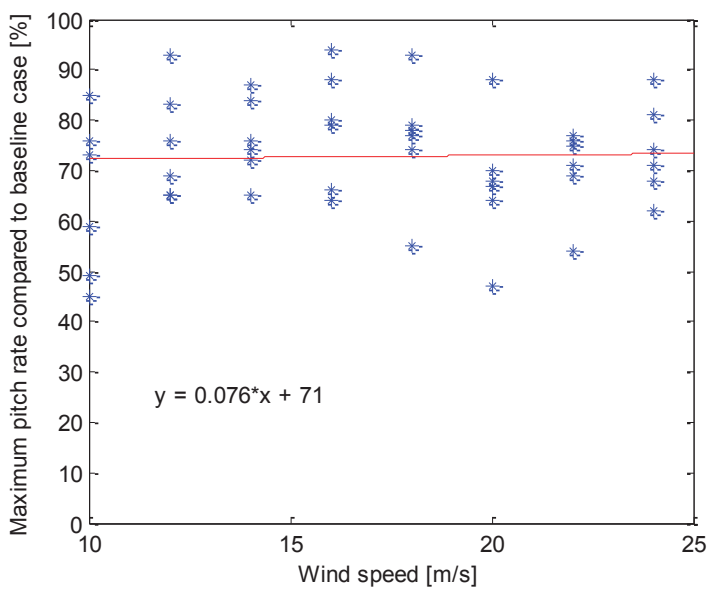

Figure 7 Maximum pitch rates compared to the baseline case

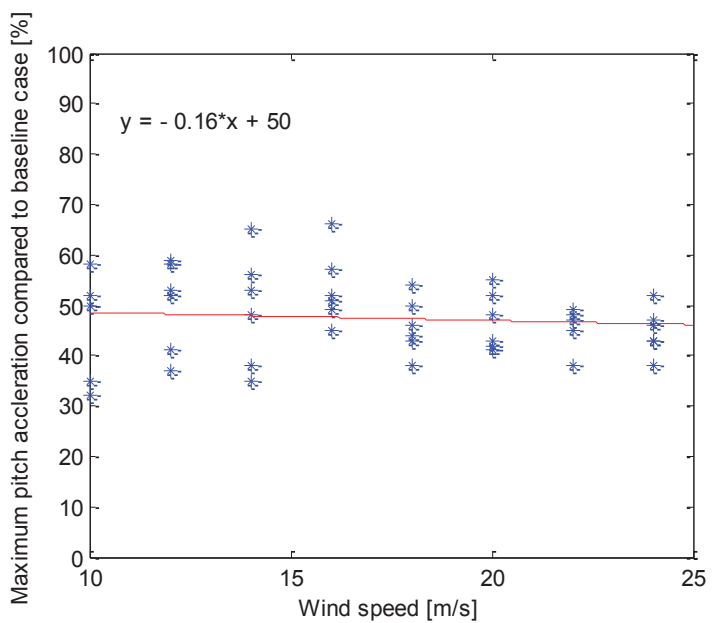

Figure 8 Maximum pitch accelerations compared to the baseline case 


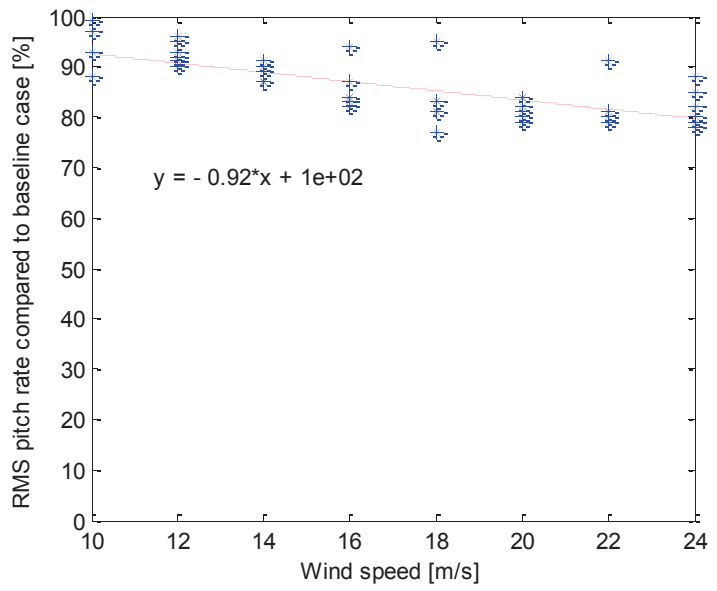

Figure 9 Root mean square (RMS) of pitch rate compared to the baseline case

\section{Consolidated smart rotor control}

A DQ-axis smart rotor control is adopted as used in [8] to assess the advantages of using the smart rotor control to both reduce loads and reduce pitch system wear. It is combined with the optimally filtered supplementary control above to form a consolidated smart rotor control strategy.

The DQ-axis control converts the rotating blade root bending moment of each blade to tilt and yaw moments in a stationary plane using the Coleman transform. The magnitude of these vectors then depict the asymmetrical yaw and tilt load components. Proportional Integral (PI) controllers then act to minimise these tilt and yaw moments, before the inverse Coleman transform is used to set the demand angle for each blade.

The DQ-axis control is set-up identically for both individual pitch and smart rotor controls, with the exception that the demand for the actuators is switched from pitch to flap angle demand and the gains increased by a factor of 8 for the smart rotor case to account for their reduced controllability. A visual representation of this strategy is shown in figure 10 .

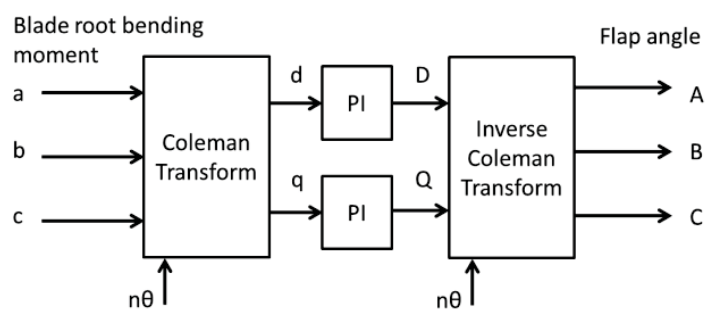

Figure 10 Smart rotor DQ-axis control load reduction strategy

Fatigue load reductions calculated with a Wohler coefficient of 4 for steel components and 10 for composite components (see [15] for details), for the blade root in-plane and out-ofplane bending moment (blade root Mx, My), the low speed shaft (rotating hub My and Mz), and the yaw bearing, are achieved using the consolidated smart rotor control. They are similar to that achieved using either Individual Pitch Control (IPC), or Smart Rotor Control operated with Collective Pitch Control (SRC + CPC), but with the added advantage that the pitch rates and accelerations are also reduced, as can be seen in table 1 . This reduction in pitch requirements varies from that when using smart rotor control for purely supplementary speed control, as described above, but the ability to both reduce loads and decrease pitch actuator requirements is attractive.

\begin{tabular}{|l|l|l|l|}
\hline Variable & IPC & SRC + CPC & SRC + sup \\
\hline Blade root Mx & $99 \%$ & $99 \%$ & $99 \%$ \\
Blade root My & $87 \%$ & $85 \%$ & $87 \%$ \\
Rotating hub My & $82 \%$ & $81 \%$ & $82 \%$ \\
Rotating hub Mz & $82 \%$ & $81 \%$ & $82 \%$ \\
Yaw bearing My & $98 \%$ & $98 \%$ & $98 \%$ \\
Yaw bearing Mz & $98 \%$ & $97 \%$ & $97 \%$ \\
Pitch rate travel & $216 \%$ & $99 \%$ & $86 \%$ \\
Pitch max rate & $169 \%$ & $107 \%$ & $65 \%$ \\
Pitch max acceleration & $119 \%$ & $96 \%$ & $91 \%$ \\
\hline
\end{tabular}

Table 1 Lifetime damage equivalent loads and pitch motion for Individual Pitch Control (IPC), Smart Rotor load reduction Control (SRC) with collective pitch control $(+\mathrm{CPC})$, and SRC with supplementary speed control (+sup) compared to the collective pitch controlled base case

The dramatic increase in pitch accelerations compared to the pure supplementary speed control case appear to be due to pitch action when switching occurs between above rated and below rated control regions, for example as seen in figure 11 which shows a particular high pitch rate and acceleration. Further work should therefore look into how to reduce the pitch demand when switching occurs between above and below rated control regions occurs.

The flap actuator has to work harder when both smart rotor load reduction control and the supplementary speed control are active, and indeed maximum deflections are increased. This may be mitigated by increasing the filter cut-off frequencies and so reduce the flap deflections contributed by the supplementary speed control, but this will impact on the pitch requirement reductions achievable as seen in the initial design study. The flap maximum deflections, rates and accelerations for the smart rotor control with collective pitch control, with supplementary control and for just supplementary speed control with no advanced load reduction control strategy are shown in table 2 .

\begin{tabular}{|l|l|l|l|}
\hline Variable & sup & SRC + CPC & SRC + sup \\
\hline Max deflection $\left({ }^{\circ}\right)$ & 9.3 & 15.2 & 16.4 \\
Max rate $\left({ }^{\circ} / \mathrm{s}\right)$ & 24 & 25.8 & 25.6 \\
Max acceleration $\left({ }^{\circ} / \mathrm{s}^{2}\right)$ & 191 & 130 & 174 \\
\hline
\end{tabular}

Table 2 Flap motion for supplementary control (sup), Smart Rotor load reduction Control (SRC) with Collective Pitch Control (+ CPC), and SRC with supplementary control (+ sup) 

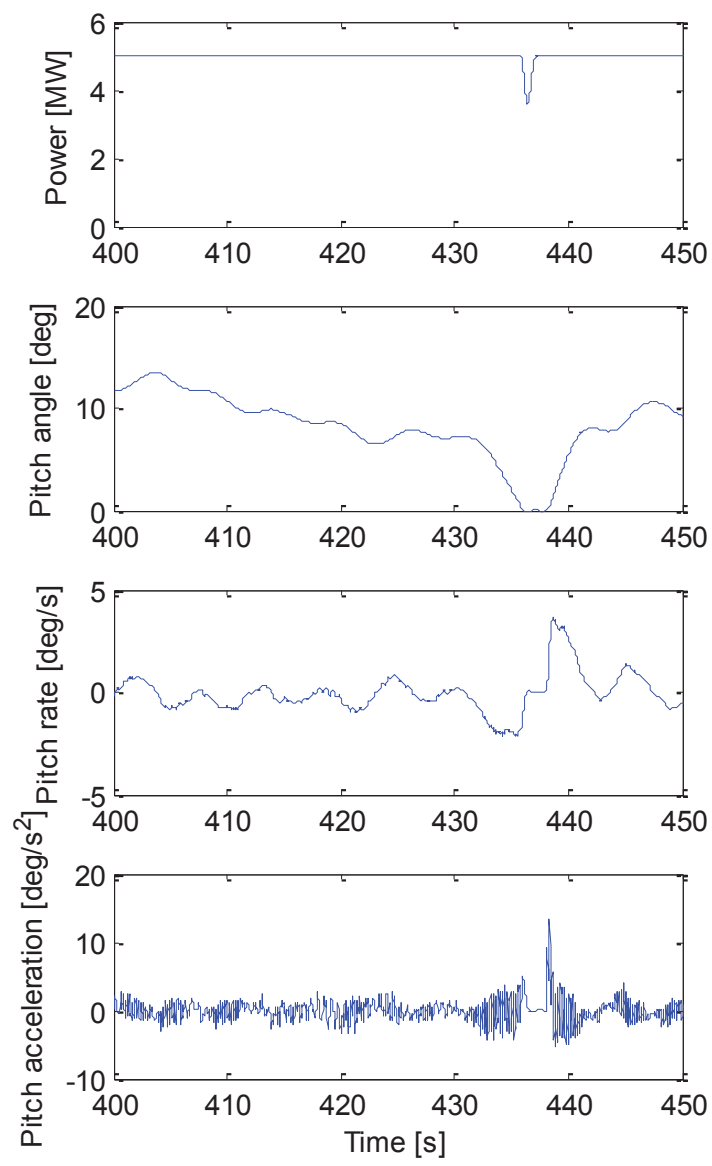

Figure 11 Time series of simulation run showing the high pitch rates and accelerations as the turbine switches between above and below rated control regions

\section{Conclusion}

While smart rotor control devices are generally not capable of fully replacing pitch control, as seen by the saturation of the flaps in this example, they can help alleviate the demands placed on the pitch actuator, as well as achieving load reductions, by supplementing the main pitch control.

Pitch travel, maximum rates and accelerations are reduced by $15 \%, 23 \%$ and $42 \%$ respectively when the smart rotor is used to assist in rotor speed control, with a trade-off between flap deflections and reduced pitch actuator demands.

Smart rotor control though is primarily about load reduction and so this supplementary speed control has been combined with a DQ-axis smart rotor control load reduction technique. This shows promising results, with reduced pitch action while still maintaining load reductions.

This demonstrates that the smart rotor control is not limited to one control objective and a trade-off should be considered between load reductions, pitch requirements, and the cost to implement and maintain the smart rotor, when considering the design of smart rotor wind turbines.

\section{Acknowledgements}

The authors would like to thank the EPSRC for funding the Wind Energy Systems Centre of Doctoral Training at the University of Strathclyde, grant number EP/G037728/1.

\section{References}

[1] "Renewables 2007: Global Status Report," REN21: Renewable Energy Policy Network for the $21^{\text {st }}$ Century, (2007)

[2] R. Wiser et al, "Wind Energy," in IPCC Special Report on Renewable Energy Sources and Climate Change Mitigation, pp. 535-608, (2011)

[3] P. Jamieson, "Innovation in Wind Turbine Design," Wiley, (2009)

[4] D. Berg et al, "The impact of active aerodynamic load control on fatigue and energy capture at low wind speed sites," European Wind Energy Conference, (2009)

[5] E. Bossanyi, "Wind Turbine Control for Load Reduction," Wind Energy, 6(3), 229-244, (2003)

[6] T. Barlas, G. van Kuik, "Review of state of the art in smart rotor control research for wind turbines," Progress in Aerospace Sciences, 46(1), pp. 1-27, (2010)

[7] S. Johnson et al., "An overview of active load control techniques for wind turbines with an emphasis on microtabs," Wind Energy, 13, 239-253, (2009)

[8] C. Plumley et al, "Comparison of individual pitch and smart rotor control strategies for load reduction," TORQUE: The Science of Making Torque from Wind, (2014)

[9] M. Lackner and G. van Kuik, "A comparison of smart rotor control approaches using trailing edge flaps and individual pitch control," Wind Energy, 13, pp. 117134, (2009)

[10] E. Bossanyi, "Bladed Theory Manual," DNV GL, Bristol, (2013)

[11] J. Jonkman, S. Butterfield, W. Musial, G. Scott, "Definition of a 5-MW Reference Wind Turbine for Offshore System Development," NREL Technical Report, (2009)

[12] J. Berg, M. Barone, N. Yoder, "SMART Wind Turbine Rotor: Data Analysis and Conclusions," Sandia Report, Sandia National Laboratories, (2014)

[13] E. Bossanyi, D. Witcher, T. Mercer, "Controller for 5MW reference turbine," Project UpWind Report, (2009)

[14] W. Leithead, S. Dominguez, "Active regulation of multi-MW wind turbines: an overview," Power Systems Technology, 31(20), pp.24-34, (2007)

[15] IEC, "IEC 61400-1 Ed.3: Wind turbines - Part 1: Design requirements," International Electro-technical Commission, (2005) 